\title{
Applying two-photon excitation fluorescence lifetime imaging microscopy to study photosynthesis in plant leaves
}

\author{
Koen Broess · Jan Willem Borst • \\ Herbert van Amerongen
}

Received: 10 December 2008/ Accepted: 27 April 2009/Published online: 26 May 2009

(C) The Author(s) 2009. This article is published with open access at Springerlink.com

\begin{abstract}
This study investigates to which extent twophoton excitation (TPE) fluorescence lifetime imaging microscopy can be applied to study picosecond fluorescence kinetics of individual chloroplasts in leaves. Using femtosecond $860 \mathrm{~nm}$ excitation pulses, fluorescence lifetimes can be measured in leaves of Arabidopsis thaliana and Alocasia wentii under excitation-annihilation free conditions, both for the $F_{0^{-}}$and the $F_{\mathrm{m}}$-state. The corresponding average lifetimes are $\sim 250 \mathrm{ps}$ and $\sim 1.5 \mathrm{~ns}$, respectively, similar to those of isolated chloroplasts. These values appear to be the same for chloroplasts in the top, middle, and bottom layer of the leaves. With the spatial resolution of $\sim 500 \mathrm{~nm}$ in the focal (xy) plane and $2 \mu \mathrm{m}$ in the $\mathrm{z}$ direction, it appears to be impossible to fully resolve the grana stacks and stroma lamellae, but variations in the fluorescence lifetimes, and thus of the composition on a pixel-to-pixel base can be observed.
\end{abstract}

Keywords Chloroplasts - Photosystem - Fluorescence . Two-photon microscopy $\cdot$ FLIM $\cdot$ Leaf

K. Broess $\cdot$ H. van Amerongen $(\bowtie)$

Laboratory of Biophysics, Wageningen University, PO Box 8128, 6700 ET Wageningen, The Netherlands

e-mail: Herbert.vanAmerongen@wur.nl

J. W. Borst · H. van Amerongen

MicroSpectroscopy Centre, Wageningen University,

6703 HA Wageningen, The Netherlands

J. W. Borst

Laboratory of Biochemistry, Wageningen University,

6703 HA Wageningen, The Netherlands

$\begin{array}{ll}\text { Abbreviations } \\ \text { TPE } & \text { Two-photon excitation } \\ \text { FLIM } & \text { Fluorescence lifetime imaging microscopy } \\ \text { Chl } & \text { Chlorophyll } \\ \text { DCMU } & \text { 3-(3,4-Dichloro-phenyl)-1,1-dimethyl-urea } \\ \text { PSII } & \text { Photosystem II } \\ \text { PSI } & \text { Photosystem I } \\ \text { LHCII } & \text { Light-harvesting complex II } \\ \text { RC } & \text { Reaction center } \\ \text { OPE } & \text { One-photon excitation } \\ \text { Car } & \text { Carotenoids }\end{array}$

\section{Introduction}

All photosynthetic organisms contain thylakoid membranes to convert solar energy into high-energy molecules such as ATP and NADPH which are used to fix carbon in energyrich molecules that are used for reproduction, growth, and maintenance. The thylakoids contain the membrane-protein complexes called photosystem I (PSI), photosystem II (PSII), cytochrome $\mathrm{b}_{6} / f$, and F-ATPase, which are the major players in oxygenic photosynthesis (Dekker and Boekema 2005; Moore et al. 1998; Nelson and Ben-Shem 2004). Both PSI and PSII contain a reaction center which is surrounded by a large "antenna", which consists of lightharvesting pigment-protein complexes. The chlorophylls (Chls) and other pigments in the antenna harvest light and transport a large part of the corresponding energy to the reaction center in which charge separation takes place. In most plants and some green algae, the thylakoid membrane is differentiated into grana stacks and stroma lamellae (Fig. 1) (Anderson 1999; Dekker and Boekema 2005; Mustárdy and Garab 2003). Other classes of photosynthetic organisms have their own unique membrane stacking 


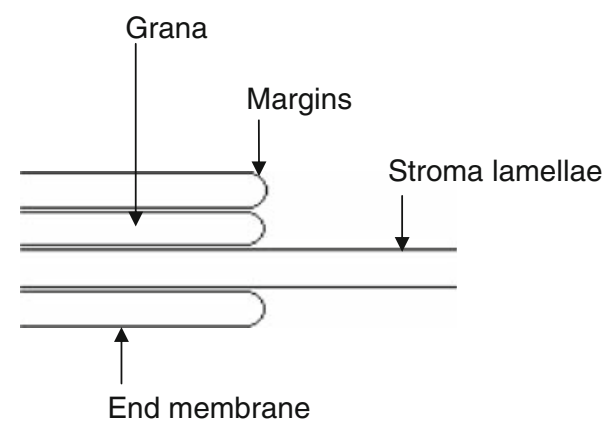

Fig. 1 Schematic model of the thylakoid membrane. The margins are the strongly curved membranes, the end membranes are located at the bottom and the top of the grana stack and the stroma lamella is the non-stacked region

which is considerably different from that of higher plants (Gunning and Schwartz 1999). The dominant antenna species of PSII in higher plants is light-harvesting complex II (LHCII) which is not only important for " "harvesting light" (van Amerongen and van Grondelle 2001), but also plays a role in nonphotochemical quenching (Pascal et al. 2005; Ruban et al. 2007), while it is, in addition, essential for grana stacking (Lambrev et al. 2007). PSI contains a large part that sticks out of the membrane and does not fit into the inner stacks of the grana. This leads to a separation of the two photosystems (Fig. 1) (Dekker and Boekema 2005). This separation is thought to allow the regulation of ATP production, by balancing the linear and cyclic electron transport (Berry and Rumberg 1996; Joliot et al. 2004) and to avoid 'spill-over'.

In higher plants about $\sim 85 \%$ of PSII is located in the grana and about $\sim 15 \%$ is present in the stroma lamellae (Fig. 1), while for PSI these numbers are approximately 35 and 65\%, respectively (Albertsson and Andreasson 2004). These percentages are not fixed but can differ between plant species while they also depend on growth conditions. However, the relative proportion of stroma lamellae and grana is rather constant (Albertsson and Andreasson 2004). The opposite is true for the number of layers in a single granum. Plants such as Alocasia that are grown in low-light intensities can have more than 50 layers in one granum, which can extend across the whole chloroplast (Goodchild et al. 1972), whereas most other plants have only $\sim 10$ till 20 layers. The diameter of the disc layer in the grana is more or less constant across plant species (300-600 nm) (Dekker and Boekema 2005; Mustárdy and Garab 2003). With the decrease of light intensity, the amount of LHCII per PSII increases, while the PSII/PSI ratio decreases. The PSII/PSI reaction centers (RCs) ratio for Alocasia, grown under low-light conditions of $10 \mu \mathrm{mol}$ photons $\mathrm{m}^{-2} \mathrm{~s}^{-1}$ is 1.43 (Chow et al. 1988). In this study, the same low-low light growing conditions are used (see Materials and Methods). The Alocasia plant was used in many chloroplast visualization studies because of its giant grana stacks (Anderson 1999; Chow et al. 1988; Goodchild et al. 1972).

The best noninvasive optical imaging technique for measuring photosynthetic systems in leaves is multiphoton fluorescence microscopy, because it allows imaging up to a depth of $500 \mu \mathrm{m}$ in living plant tissue (Williams et al. 2001; Zipfel et al. 2003). The leaves of Arabidopsis thaliana and Alocasia wentii are 200 and $300 \mu \mathrm{m}$ thick, respectively, and in principle, suitable for complete scanning by FLIM with two-photon excitation (TPE) at $860 \mathrm{~nm}$. In contrast, one-photon excitation (OPE) microscopy only allows imaging up to a depth of $\sim 100 \mu \mathrm{m}$ (Cheong et al. 1990; Williams et al. 2001). Two-photon (nonlinear) microscopy depends on the simultaneous interaction of two photons with a molecule, resulting in a quadratic dependence of light absorption on light intensity as opposed to the linear dependence of one-photon fluorescence microscopy. For pigment molecules such as chlorophylls (Chl) and carotenoids (Car), the two-photon absorption spectra, which are only partly known, are significantly different from their one-photon counterparts, but the emission spectra are in general identical ( $\mathrm{Xu}$ et al. 1996). For LHCII, the TPE spectrum was measured in the region from 1,000 to $1,600 \mathrm{~nm}$, "“corresponding", to onephoton wavelengths of $500-800 \mathrm{~nm}$ (Walla et al. 2000).

This study combines microscopy with fluorescence lifetime measurements to investigate to which extent it is possible to study the primary steps in photosynthesis in living tissue and to determine at which spatial and time resolution this is possible. The final goal is to study these primary events in vivo under a variety of (stress) conditions.

In this study, the two-photon absorption of $860 \mathrm{~nm}$ light is used for excitation. The instrument response function (IRF) of the FLIM setup is $25 \mathrm{ps}$ (van Oort et al. 2008). Because carotenoids and Chl $b$ transfer most of their excitation energy to Chl $a$ in less than $1 \mathrm{ps}$ (Croce et al. 2001, 2003; Eads et al. 1989; Gradinaru et al. 2000; Peterman et al. 1997; van Amerongen and van Grondelle 2001; Visser et al. 1996) only fluorescence from Chl $a$ is observed (Broess et al. 2008). We focus on the detection of fluorescence lifetimes of Chl in PSI and PSII in intact leaves, both under low-light conditions and under conditions in which the PSII reaction centers are closed by DCMU. The shade plant Alocasia wentii is used to make it easier to try to spatially resolve the different photosystems, because it is known to form giant grana stacks (Albertsson and Andreasson 2004; Goodchild et al. 1972). In addition, Arabidopsis thaliana is studied because it is widely used as one of the model organisms in plant sciences. 


\section{Materials and methods}

Fluorescence lifetime imaging microscopy

Multiphoton imaging was performed on a multiphoton dedicated Biorad Radiance 2100 MP system, coupled to a Nikon TE300 inverted microscope (Borst et al. 2003). A tunable Ti-Sapphire laser (Coherent Mira) was used as an excitation source which was pumped with a 5-Watt (Coherent) Verdi laser, resulting in excitation pulses of $\sim 200 \mathrm{fs}$ at a repetition rate of $76 \mathrm{MHz}$. In the beamconditioning unit (BCU), the excitation power was tuned by a pockell cell. The laser beam was collimated in the scanhead and focused by a Nikon 60x water immersion Apochromat objective lens (NA 1.2) into the sample. The fluorescence was detected by non-descanned direct detectors (NDDs), which were coupled to the sideport of the microscope. Using this type of detection, the loss of fluorescence light was reduced, and 3-5 times more signal was obtained compared to internal detectors. The emission light was split into two channels using a dichroic mirror filter wheel. FLIM measurements were performed by directing the fluorescence via a secondary dichroic (770DCXR, Chroma Technology Corp.) into a Hamamatsu R3809U photomultiplier, operated at $3.1 \mathrm{kV}$. Fluorescence was selected using a dichroic (FF 495- $\mathrm{DiO}_{2}$, Semrock) and 2x a bandpass filter (HQ700/75, Chroma Technology Corp). In the excitation branch, a longpass filter (RG $7803 \mathrm{~mm}$, Schott) was used for reduction of the excitation light.

The multichannel-plate photomultiplier allows single photon detection at high time-resolution, with an IRF of 25 ps (van Oort et al. 2008, 2009). The output of the detector was coupled to a Becker \& Hickl single-photoncounting module (SPC 830) (Becker and Bergmann 2002). The signal from the Hamamatsu triggers the START of the time ramping for the time-correlated single-photon-counting (TCSPC). The pulses from the TiSapphire laser serve as the SYNC signal to stop the time ramping and allowing the timing of the arrival of the fluorescent photons. The time window (ADC) was set to 1,024 channels and typically fluorescence was recorded for $2 \mathrm{~min}$ at a photon count rate of approximately $20 \mathrm{kHz}$. The signal from the PMT is combined with the pixel clock and line predivider signals from the Biorad scanhead to create 2D lifetime images. Fluorescence decay curves were fitted to a sum of $N$ exponentials $\Sigma \mathrm{a}_{\mathrm{i}} \exp \left(-\tau / \tau_{i}\right)$ ( $i$ runs from 1 to $N$ ), convoluted with the IRF (Digris et al. 1999, van Oort et al. 2008, 2009), which was determined from the decay of pinacyanol iodide in methanol. From these results, an average lifetime $\langle\tau\rangle$ was also calculated, according to $\left\langle\tau>=\Sigma a_{i} \tau_{i}\right.$. The number of counts in the peak channels is $\sim 100$ in the fluorescence intensities images and traces.
Growth condition and sample preparation

Arabidopsis was grown in growth chambers of $23^{\circ} \mathrm{C}$ with alternating $16 \mathrm{~h}$ of light at a light intensity of 60$100 \mu \mathrm{E} \mathrm{m}^{-2} \mathrm{~s}^{-1}$ and $8 \mathrm{~h}$ of darkness. Note that the light intensity is relatively low, which may lead to an altered antenna composition as compared to that of plants grown under high-light conditions. Alocasia was grown at room temperature with alternating $16 \mathrm{~h}$ of light at a light intensity of $10-15 \mu \mathrm{E} \mathrm{m}^{-2} \mathrm{~s}^{-1}$ and $8 \mathrm{~h}$ of darkness. For closing the reaction centers of PSII in leaves, vacuum infiltration was performed with $0.1 \mathrm{mM}$ DCMU, $20 \mathrm{mM}$ Hepes, $5 \mathrm{mM} \mathrm{NaCl}$, and $5 \mathrm{mM} \mathrm{MgCl} 2$ buffer with $\mathrm{pH}$ 7.5.

Isolation of chloroplasts: Alocasia wentii leaves were ground in semi-frozen buffer $1(0.45 \mathrm{M}$ sorbitol, $20 \mathrm{mM}$ Tricine, $10 \mathrm{mM}$ EDTA, $10 \mathrm{mM} \mathrm{NaHCO}$, and $0.1 \%$ BSA, $\mathrm{pH}$ 8.4) using a blender for 5-s, and then filtrated through eight layers of cheesecloth and centrifuged at 3,000 $\times g$ for $20 \mathrm{~s}$ at $4{ }^{\circ} \mathrm{C}$. The supernatant was discarded and the pellet washed with buffer $2(0.3 \mathrm{M}$ Sorbitol, $20 \mathrm{mM}$ Tricine, $5 \mathrm{mM} \mathrm{MgCl}_{2}$, and $2.1 \mathrm{mM}$ EDTA, pH 7.4). The collected resuspended chloroplasts were put on $50 \%$ Percoll $/ 50 \%$ buffer 3 (0.6 M Sorbitol, $20 \mathrm{mM}$ Tricine, and $5 \mathrm{mM} \mathrm{MgCl}_{2}, \mathrm{pH}$ 7.6) and centrifuged at 3,500 $\times \mathrm{g}$ for $10 \mathrm{~min}$ at $4^{\circ} \mathrm{C}$. The supernatant was disposed, and the pellet was diluted in buffer 2 before measuring.

\section{Results and discussion}

It has been demonstrated that FLIM can be a noninvasive tool (Holub et al. 2000; Lukins et al. 2005) for measuring Chl $a$ fluorescence lifetimes in plants and algae which can then be correlated to the response of the photosynthetic apparatus to, for instance, the effect of dehydration. However, measurements so far have only been performed under high-light conditions at the maximum fluorescence level $\left(\mathrm{F}_{\mathrm{M}}\right)$ in which PSII reaction centers are closed and average lifetimes were found to be $1.7 \mathrm{~ns} \pm 0.2 \mathrm{~ns}$ (Holub et al. 2000) and 611 ps (Lukins et al. 2005), indeed corresponding to lifetimes of PSII with (partially) closed reaction centers.

With the FLIM setup used in the present study it is possible to measure under low-light conditions. In Fig. 2 two images with 1,024 pixels are presented, showing Alocasia wentii chloroplasts. The fluorescence images illustrated in the figure are intensity-based, whereas the fluorescence kinetics has been obtained for each pixel and has been analyzed with a combination of SPCimage2.3 software (Becker \& Hickl) and home-built software using a exponential decay model (Digris et al. 1999; Mullen et al. 2007; Novikov et al. 1999). The fitted chloroplast fluorescence lifetimes and amplitudes averaged over all the 


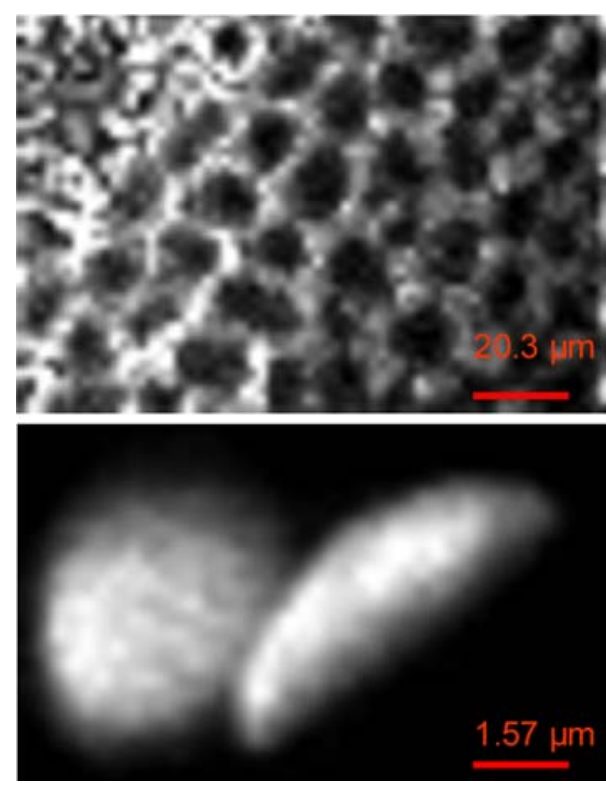

Fig. 2 Room temperature fluorescence intensity-based image $(1,024$ pixels) with a linear gray scale as measured with FLIM. The chloroplasts in Alacosia wentii leaves are excited with TPE at $860 \mathrm{~nm}$ and detected with a bandpass filter centered at $700 \mathrm{~nm}$ and with a bandwidth of $75 \mathrm{~nm}$. For each pixel a fluorescence decay trace is measured. The average lifetimes and amplitudes in the 1,024 pixels in this image are: $\tau_{1} 59.5 \mathrm{ps}(44.1 \%), \tau_{2} 205 \mathrm{ps}(35.3 \%)$ and $\tau_{3} 588 \mathrm{ps}$ $(20.6 \%)$

pixels of Fig. $2 \mathrm{~b}$ are as follows: $\tau_{1}=59.5 \mathrm{ps}(44.1 \%)$, $\tau_{2}=205 \mathrm{ps}(35.3 \%)$ and $\tau_{3}=588 \mathrm{ps}(20.6 \%)$. Without further experiments and knowledge, it is not possible to assign the lifetimes to processes in PSI and PSII. The amplitudes are expected to depend strongly on the excitation and detection wavelength. A complicating factor at this stage is the fact that the two-photon absorption spectra of all the relevant pigments are not known. Scanning at different depths in the leaf does not result in different average lifetimes or different amplitudes. Figure $2 a$ and $b$ are intensity-based images with a linear gray scale. Pixels with zero fluorescence counts are dark and pixels with maximum fluorescence are white. The chloroplasts in Fig. 2b appear to be heterogeneous, and small white dots can be observed within the chloroplast. Similar heterogeneity was observed earlier in microscope measurements (Anderson 1999; Spencer and Wildman 1962; van Spronsen et al. 1989), and it is likely that the white spots correspond to the grana stacks. The Chl concentration is higher in the grana and moreover, as they contain mainly PSII, which leads to more fluorescence than PSI because of the longer fluorescence lifetimes.

It is known from TPE FLIM measurements on LHCII aggregates (Barzda et al. 2001a) that the pulse repetition rates of more than $1 \mathrm{MHz}$ can lead to the shortening of fluorescence lifetimes of photosynthetic systems because of

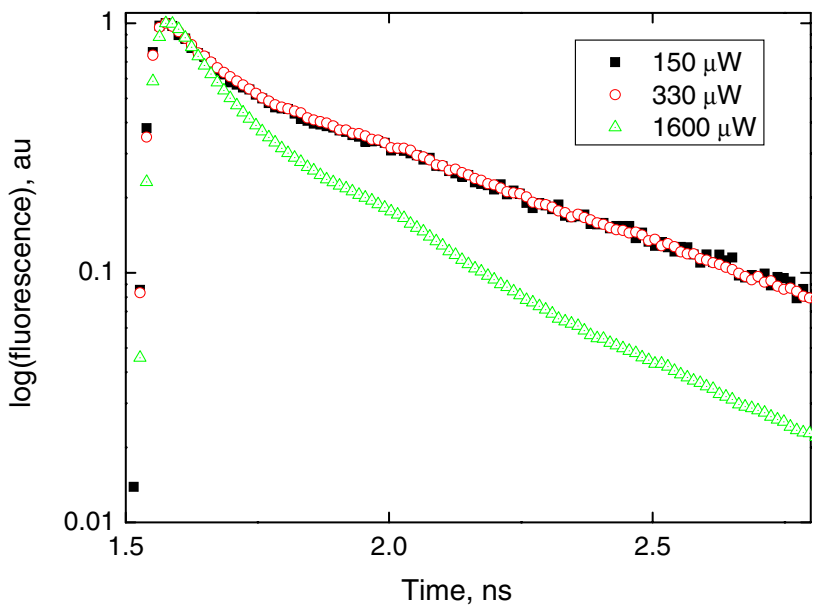

Fig. 3 Room temperature fluorescence decay traces (measured with FLIM) of chloroplasts in Arabidopsis thaliana leaves. The chloroplasts are excited with TPE at $860 \mathrm{~nm}$ and detected with a bandpass filter centered at $700 \mathrm{~nm}$ with a bandwidth of $75 \mathrm{~nm}$. Identical traces are observed for chloroplasts with laser powers of $150 \mu \mathrm{W}$ (black squares) and $330 \mu \mathrm{W}$ (red open circles) and correspond to PSII with open reaction centers. The green open triangles represent a decay trace obtained with a laser power of $1,600 \mu \mathrm{W}$ which is faster than the black and red decay traces due to annihilation processes. The number of counts in the peak channels are 28,156 , and 2028 , respectively

excitation quenching by Car and Chl triplets (singlet-triplet annihilation). Moreover, singlet-singlet annihilation can occur, also leading to a shortening of the lifetime (Barzda et al. 2001b). Since the number of triplets formed is expected to increase on increasing the number of excitations, the fluorescence lifetimes have been measured as a function of laser intensity. In Fig. 3, three decay traces are presented, obtained at 150,330 , and $1600 \mu \mathrm{W}$ (laser power measured directly at the sample holder of the setup). The 150 and $330 \mu \mathrm{W}$ decay traces are identical after normalization at the top, whereas the $1,600 \mu \mathrm{W}$ trace is substantially faster. It should be noted that the initial number of excitations for TPE scales quadratically with the light intensity, and thus the number of excitations increases by a factor of 4.8 when going from 150 to $330 \mu \mathrm{W}$. Therefore, the results clearly demonstrate the absence of singlettriplet (and singlet-singlet) annihilation at relatively low intensities. Using extremely high powers of $1,600 \mu \mathrm{W}$, the $\mathrm{RCs}$ are being closed, but the kinetics are faster, which is ascribed to a combination of singlet-singlet and singlettriplet annihilation.

The fluorescence decay traces of isolated chloroplasts have also been measured with FLIM and are compared to those of leaf tissue (Fig. 4). The in vivo fluorescence kinetics of chloroplasts are similar to those of the isolated chloroplasts for the first 170-ps part of the trace. There is a small discrepancy in the middle part of the trace, but overall the traces are nearly identical. The chloroplasts 


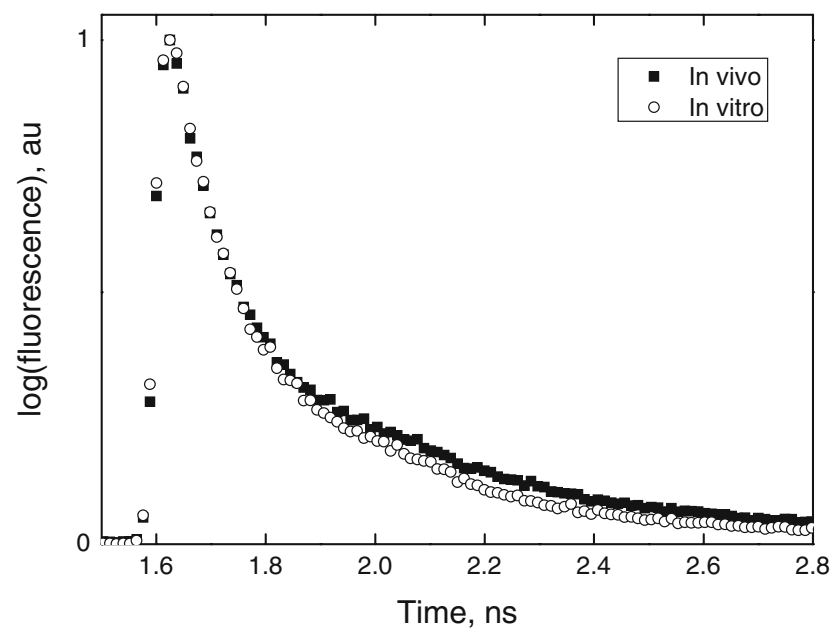

Fig. 4 Room temperature fluorescence decay traces (measured with FLIM). The chloroplasts in Alocasia wentii are excited with TPE at $860 \mathrm{~nm}$ and detected with a bandpass filter centered at $700 \mathrm{~nm}$ with a bandwidth of $75 \mathrm{~nm}$. Round open circles are isolated chloroplasts (in vitro) with an average lifetime of $180 \mathrm{ps}$. Black squares correspond to chloroplasts in leaves (in vivo) with an average lifetime of $212 \mathrm{ps}$

were isolated with percoll and are smaller in size (not shown) than the chloroplasts in leaves.

In order to try to distinguish between PSI and PSII in the microscopic images, the difference in fluorescence lifetimes between the two photosystems has been increased by closing the reaction centers of PSII by vacuum infiltration of Arabidopsis thaliana with $0.1 \mathrm{mM}$ DCMU in $20 \mathrm{mM}$ Hepes, $5 \mathrm{mM} \mathrm{NaCl}$, and $5 \mathrm{mM} \mathrm{MgCl} 2$ buffer with $\mathrm{pH}$ 7.5. The average lifetime for the leaf infiltrated with DCMU is $1.3 \mathrm{~ns}$ (Fig. 5) whereas for "normal" leaves the average lifetime is 290 ps. Both photosystems are separated in space and have substantially different lifetimes in the presence of DCMU (Lukins et al. 2005; Pfündel 1998; Zucchelli et al. 1992) because the average lifetime of PSI with antenna complexes is reported to be $\sim 60$ ps (Croce et al. 2000; van Oort et al. 2008) and that of closed PSII is $\sim 1.5 \mathrm{~ns}$ (Zucchelli et al. 1992). This is visible in the traces and images of the chloroplasts of Alocasia wentii in Fig. 6. The expectation is that pixels with more grana stacks have a higher intensity compared to pixels with relatively more stroma lamellae (Spencer and Wildman 1962). In Fig. 6a, the fluorescence kinetics of 10 high-intensity pixels (white) are compared with those of 10 low-intensity pixels (grey). The 10 highand low-intensity pixels have $623(266,342)$ and 541 $(195,833)$ counts in the peak (and total number of fluorescence counts), respectively. The global fitting results with linked lifetimes and independent amplitudes are $\tau_{1}=116$ ps $(53.3,59.6 \%), \tau_{2}=1,027$ ps $(35.1,29.5 \%)$, and $\tau_{3}=3,957 \mathrm{ps}(11.6,10.9 \%)$. The first amplitude for each lifetime refers to the high-intensity pixels and the

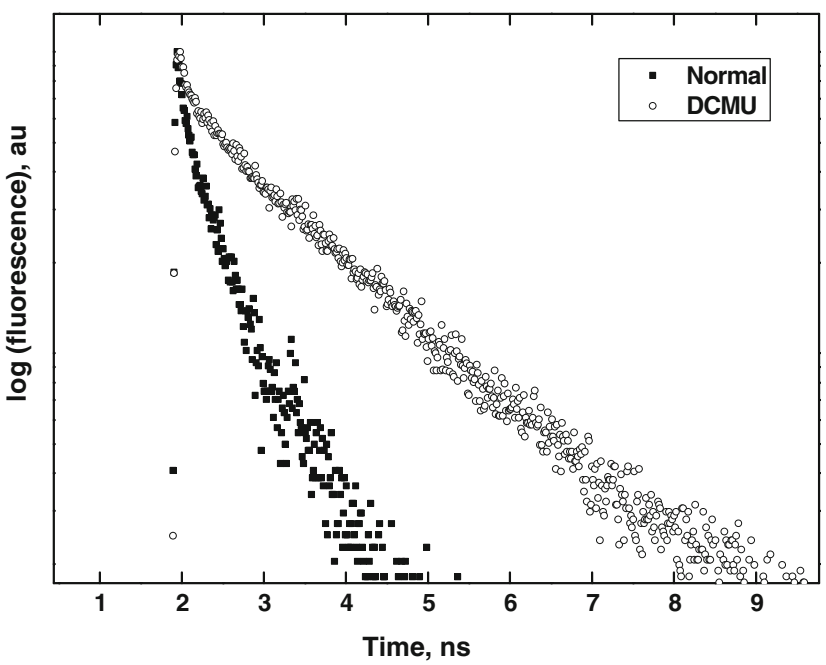

Fig. 5 Room temperature fluorescence decay traces (measured with FLIM). The chloroplasts in Arabidopsis thaliana leaves are excited with TPE at $860 \mathrm{~nm}$ and are detected with a bandpass filter centered at $700 \mathrm{~nm}$ with a bandwidth of $75 \mathrm{~nm}$. Black squares represent a "normal" fluorescence decay trace of chloroplasts in an Arabidopsis leaf with an average lifetime of 290 ps. Round open circles represent a fluorescence decay trace of a vacuum infiltrated leaf with a $0.1 \mathrm{mM}$ DCMU buffer with an average lifetime of $1.3 \mathrm{~ns}$

second amplitude, to the low-intensity pixels. The first lifetime of 116 ps probably reflects a mixture of PSI and open PSII reaction centers (Broess et al. 2006, 2008) because Alocasia grown under low-light conditions have a PSII/PSI RCs ratio of 1.43 (Chow et al. 1988). This corresponds to $59 \% \mathrm{Chl}$ of PSII and $41 \% \mathrm{Chl}$ of PSI. If all the PSIIs are closed, one might expect $59 \% \mathrm{Chl}$ contribution of slow lifetimes and $41 \%$ of fast lifetime. The amplitudes of the lifetime of $116 \mathrm{ps}$ for both groups of pixels is more than $41 \%$, so the conclusion should be such that not all the PSII reaction centers are closed by the DCMU. The two slow lifetimes of $\sim 1$ and $\sim 4$ ns must correspond to closed PSII reaction centers because these lifetimes are absent for open RCs. The $6.3 \%$ difference in the amplitude of the slow lifetimes for the high- and low-intensity pixels is probably caused by the fact that the high-intensity pixels comprise more PSII than PSI. This is expected because the grana, where PSII is concentrated, have a higher chlorophyll concentration per pixel than the stroma lamellae. There are two straightforward explanations for the lifetime differences in the pixel groups: (i) The DCMU buffer is not penetrated evenly in every part of the chloroplasts which results in different lifetimes and intensities for each pixel; (ii) In one pixel group, there are more grana than in the other pixel group which will also result in different lifetimes and intensities for each pixel. In Fig. 6b, the intensity of the different pixels seems to have a random distribution in the chloroplast, which is not expected as a result of varying 
(a)

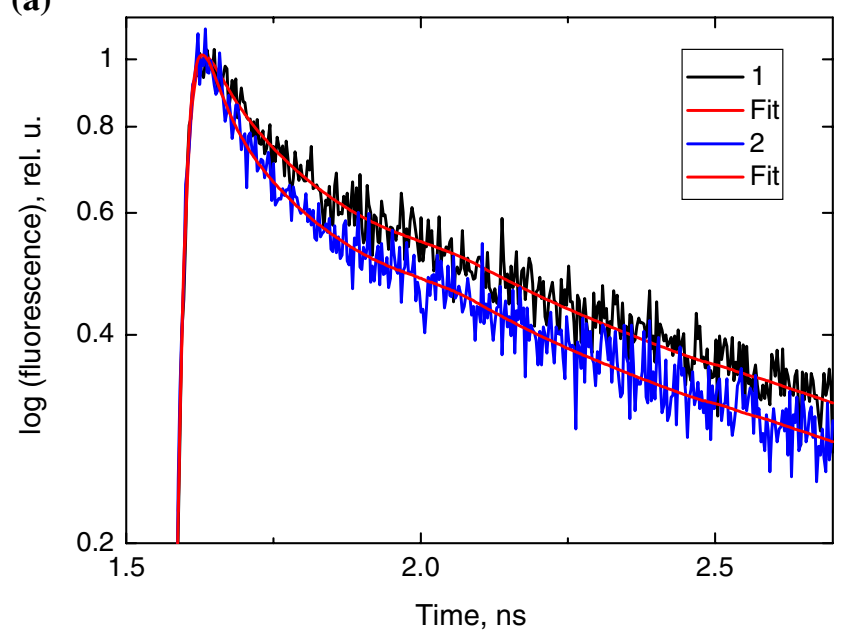

(b)

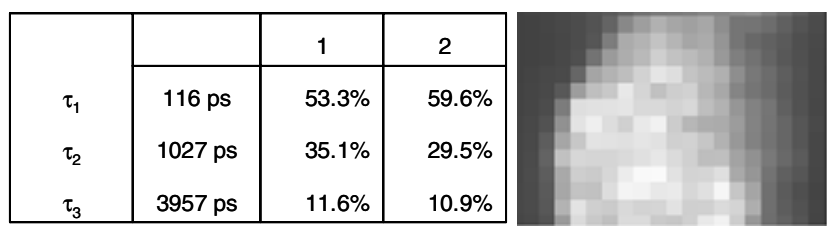

Fig. 6 a Room temperature fluorescence decay traces (measured with FLIM) of chloroplasts in Alocasia wentii leaves excited with TPE at $860 \mathrm{~nm}$ detected with a bandpass filter centered at $700 \mathrm{~nm}$ with a bandwidth of $75 \mathrm{~nm}$. The leaves are vacuum infiltrated with a $0.1 \mathrm{mM}$ DCMU buffer for closing the PSII reaction centers. The black (1) trace with its fit corresponds to the summed fluorescence decay of 10 white (high) pixels from the chloroplast in the intensitybased image in Fig. 6b. The blue (2) trace with its fit corresponds to the summed fluorescence decay of 10 grey (low) pixels from the chloroplast in the intensity-based image in Fig. 6b. b (right) Room temperature fluorescence intensity-based image (measured with FLIM). The chloroplast in Alacosia wentii leaves are excited with TPE at $860 \mathrm{~nm}$ and detected with a bandpass filter centered at $700 \mathrm{~nm}$ with a bandwidth of $75 \mathrm{~nm}$. The pixel size is $0.26 \mu \mathrm{m}$. Fluorescence in each pixel is detected in 4,096 channels with a time resolution of 3 ps per channel. (left) Global fitting with linked lifetimes $\left(\tau_{1}, \tau_{2}\right.$, and $\tau_{3}$ ) and independent amplitudes for the black trace (1) and blue trace (2) shown in Fig. 6a

penetration of the DCMU buffer. The differences in lifetimes for the two pixel groups can thus better be explained by pixels with more or less grana. It should be kept in mind that the model that is used here (PSI and PSII fluorescence kinetics are both homogeneous) is oversimplified, for instance, because of the action of the PSII repair cycle and the presence of PSII heterogeneity. In conclusion, it appears to be very difficult to distinguish between regions with more or less grana.

For Arabidopsis thaliana leaves, it appears to be not at all possible to resolve variations in lifetimes between pixels, which is probably due to the fact that for Arabidopsis thaliana, the grana stacks are smaller than for Alocasia wentii.

\section{Conclusions}

- In vivo measurements on chloroplasts are possible under low-light conditions with TPE FLIM and the obtained fluorescence kinetics are very similar to those observed in in vitro measurements on isolated chloroplasts.

- While scanning through the leaves of Alocasia wentii and Arabidopsis thaliana that were both grown under low-light conditions, no differences could be observed in the fluorescence kinetics, indicating no variation in the chloroplast composition/organization as a function of depth.

- The spatial resolution of the FLIM measurements does not allow to observe individual grana stacks in Arabidopsis thaliana chloroplasts, but in the case of chloroplasts of Alocasia wentii variations in the lifetimes are observed, which may be ascribed to variations in the grana density.

- In the future, the TPE fluorescence lifetime imaging microscope can be used to study individual chloroplasts in leaves under different stress conditions.

Acknowledgments This study is part of the research programme of the "Stichting voor Fundamenteel Onderzoek der Materie (FOM)," which is financially supported by the "Nederlandse Organisatie voor Wetenschappelijk Onderzoek (NWO).”

Open Access This article is distributed under the terms of the Creative Commons Attribution Noncommercial License which permits any noncommercial use, distribution, and reproduction in any medium, provided the original author(s) and source are credited.

\section{References}

Albertsson PÄ, Andreasson E (2004) The constant proportion of grana and stroma lamellae in plant chloroplasts. Physiol Plant 121:334-342. doi:10.1111/j.0031-9317.2004.00315.x

Anderson JM (1999) Insights into the consequences of grana stacking of thylakoids membranes in vascular plants: a personal perspective. Aust J Plant Physiol 26:625-639

Barzda V, de Grauw CJ, Vroom J, Kleima FJ, van Grondelle R, van Amerongen H, Gerritsen HC (2001a) Fluorescence lifetime heterogeneity in aggregates of LHCII revealed by time-resolved microscopy. Biophys J 81:538-546. doi:10.1016/S0006-3495 (01) $75720-7$

Barzda V, Gulbinas V, Kananavicius R, Cervinskas V, van Amerongen H, van Grondelle R, Valkunas L (2001b) Singlet-singlet annihilation kinetics in aggregates and trimers of LHCII. Biophys J 80:2409-2421. doi:10.1016/S0006-3495(01)76210-8

Becker W, Bergmann A (2002) Lifetime imaging techniques for optical microscopy. Becker and Hickl GmbH, Berlin

Berry S, Rumberg B (1996) $\mathrm{H}^{+} /$ATP coupling ratio at the unmodulated CF0CF1-ATP synthase determined by proton flux measurements. Biochim Biophys Acta 1276:51-56

Borst JW, Hink MA, Van Hoek A, Visser AJWG (2003) Multiphoton microspectroscopy in living plant cells. Proc SPIE 4963:231238. doi: $10.1117 / 12.477989$ 
Broess K, Trinkunas G, van der Weij-de Wit CD, Dekker JP, van Hoek A, van Amerongen H (2006) Excitation energy transfer and charge separation in photosystem II membranes revisited. Biophys J 91:3776-3786. doi:10.1529/biophysj.106.085068

Broess K, Trinkunas G, van Hoek A, Croce R, van Amerongen H (2008) Determination of the excitation migration time in photosystem II: Consequences for the membrane organization and charge separation parameters. Biochim Biophys Acta 1777: 404-409

Cheong WF, Prahl SA, Welch AJ (1990) A review of the optical properties of biological tissues. IEEE J Quantum Electron 26:2166-2185. doi:10.1109/3.64354

Chow WS, Anderson JM, Hope AB (1988) Variable stoichiometries of photosystem II to photosystem I reaction centres. Photosynth Res 17:277-281. doi:10.1007/BF00035454

Croce R, Dorra D, Holzwarth AR, Jennings RC (2000) Fluorescence decay and spectral evolution in intact photosystem I of higher plants. Biochemistry 39:6341-6348. doi:10.1021/bi992659r

Croce R, Muller MG, Bassi R, Holzwarth AR (2001) Carotenoid-tochlorophyll energy transfer in recombinant major light-harvesting complex (LHCII) of higher plants. I. Femtosecond transient absorpt measurements. Biophys J 80:901-915

Croce R, Muller MG, Bassi R, Holzwarth AR (2003) Chlorophyll b to chlorophyll a energy transfer kinetics in the CP29 antenna complex: a comparative femtosecond absorption study between native and reconstituted proteins. Biophys J 84:2508-2516. doi: 10.1016/S0006-3495(03)75056-5

Dekker JP, Boekema EJ (2005) Supramolecular organization of thylakoid membrane proteins in green plants. Biochim Biophys Acta 1706:12-39. doi:10.1016/j.bbabio.2004.09.009

Digris AV, Skakoun VV, Novikov EG, Van Hoek A, Claiborne A, Visser AJWG (1999) Thermal stability of a flavoprotein assessed from associative analysis of polarized time-resolved fluorescence spectroscopy. Eur Biophys J 28:526-531. doi:10.1007/ s002490050235

Eads DD, Castner EW, Alberte RS, Mets L, Fleming GR (1989) Direct observation of energy transfer in a photosynthetic membrane: chlorophyll b to chlorophyll a transfer in LHC. J Phys Chem 93:8271-8275. doi:10.1021/j100363a001

Goodchild DJ, Björkman O, Pyliotis NA (1972) Chloroplast ultrastructure, leaf anatomy, and content of chlorophyll and soluble protein in rainforest species. Carnegie Inst Wash Yearb 71:102-107

Gradinaru CC, van Stokkum IHM, Pascal AA, van Grondelle R, van Amerongen $\mathrm{H}$ (2000) Identifying the pathways of energy transfer between carotenoids and chlorophylls in LHCII and CP29. A multicolor, femtosecond pump-probe study. J Phys Chem B 104:9330-9342. doi:10.1021/jp001752i

Gunning BES, Schwartz OM (1999) Confocal microscopy of thylakoid autofluorescence in relation of grana and phylogeny in the green algae. Aust J Plant Physiol 26:695-708

Holub O, Seufferheld MJ, Gohlke C, Govindjee, Clegg RM (2000) Fluorescence lifetime imaging (FLI) in real time-a new technique in photosynthesis research. Photosynthetica 38:581599. doi:10.1023/A:1012465508465

Joliot P, Béal D, Joliot A (2004) Cyclic electron flow under saturating excitation of dark-adapted Arabidopsis leaves. Biochim Biophys Acta 1656:166-176

Lambrev PH, Várkonyi Z, Krumova S, Kovács L, Miloslavina Y, Holzwarth AR, Garab G (2007) Importance of trimer-trimer interactions for the native state of the plant light-harvesting complex II. Biochim Biophys Acta 1767:847-853

Lukins PB, Rehman S, Stevens GB, George D (2005) Time-resolved spectroscopic fluorescence imaging, transient absorption and vibrational spectroscopy of intact and photo-inhibited photosynthetic tissue. Luminescence 20:143-151. doi:10.1002/bio.819
Moore R, Clark WD, Vodopich DS (1998) Botany. Bios Scientific Publishers, Springer-Verlag, New York and WCB McGraw-Hill, Dubuque, 919 pp. ISBN 0-69728623-1

Mullen KM, van Stokkum IHM, Laptenok S, Borst JW, Apanasovich VV, Visser AJWG (2007) Fluorescence lifetime imaging microscopy (FLIM) data analysis with TIMP. J Stat Softw 18: $1-20$

Mustárdy L, Garab G (2003) Granum revisited. A three-dimensional model-where things fall into place. Trends Plant Sci 8:117122. doi:10.1016/S1360-1385(03)00015-3

Nelson N, Ben-Shem A (2004) The complex architecture of oxygenic photosynthesis. Nat Rev Mol Cell Biol 5:971-982. doi:10.1038/ nrm 1525

Novikov EG, van Hoek A, Visser AJWG, HJ W (1999) Linear algorithms for stretched exponential decay analysis. Opt Commun 166:189-198. doi:10.1016/S0030-4018(99)00262-X

Pascal AA, Liu Z, Broess K, van Oort B, van Amerongen H, Wang C, Horton P, Robert B, Chang W, Ruban A (2005) Molecular basis of photoprotection and control of photosynthetic light-harvesting. Nature 436:134-137. doi:10.1038/nature03795

Peterman EJG, Monshouwer R, van Stokkum IHM, van Grondelle R, van Amerongen $H$ (1997) Ultrafast singlet excitation transfer from carotenoids to chlorophylls via different pathways in lightharvesting complex II of higher plants. Chem Phys Lett 264: 279-284. doi:10.1016/S0009-2614(96)01334-6

Pfündel E (1998) Estimating the contribution of photosystem I to total leaf chlorophyll fluorescence. Photosynth Res 56:185-195. doi: 10.1023/A:1006032804606

Ruban AV, Berera R, Ilioaia C, van Stokkum IHM, Kennis JTM, Pascal AA, van Amerongen $\mathrm{H}$, Robert B, Horton $\mathrm{P}$, van Grondelle R (2007) Identification of a mechanism of photoprotective energy dissipation in higher plants. Nature 450:575-578. doi:10.1038/nature06262

Spencer D, Wildman SG (1962) Observations on structure of granacontaining chloroplast and a proposed model of chloroplast structure. Aust J Biol Sci 15:599-610

van Amerongen H, van Grondelle R (2001) Understanding the energy transfer function of LHCII, the major light-harvesting complex of green plants. J Phys Chem B 105:604-617. doi:10.1021/ jp0028406

van Oort B, Amunts A, Borst JW, van Hoek A, Nelson N, van Amerongen $\mathrm{H}$ (2008) Picosecond fluorescence of intact and dissolved PSI-LHCI crystals. Biophys J 95:5851-5861. doi:10. 1529/biophysj.108.140467

van Oort B, Murali S, Wientjes E, Koehorst RBM, Spruijt RB, van Hoek A, Croce R, van Amerongen H (2009) Ultrafast resonance energy transfer from a site-specifically attached fluorescent chromophore reveals the folding of the N-terminal domain of CP29. Chem Phys 357:113-119. doi:10.1016/j.chemphys.2008. 10.052

van Spronsen EA, Sarafis V, Brakenhoff GJ, van der Voort HTM, Nanninga N (1989) Three-dimensional structure of living chloroplasts as visualized by confocal scanning laser microscopy. Protoplasma 148:8-14. doi:10.1007/BF01403986

Visser HM, Kleima FJ, van Stokkum IHM, van Grondelle R, van Amerongen $\mathrm{H}$ (1996) Probing the many energy-transfer processes in the photosynthetic light-harvesting complex II at $77 \mathrm{~K}$ using energy-selective sub-picosecond transient absorption spectroscopy. Chem Phys 210:297-312. doi:10.1016/0301-0104 (96)00092-4

Walla PJ, Yom J, Krueger BP, Fleming GR (2000) Two-photon excitation spectrum of light-harvesting complex II and fluorescence upconversion after one- and two-photon excitation of the carotenoids. J Phys Chem B 104:4799-4806. doi:10.1021/ jp9943023 
Williams RM, Zipfel WR, Webb WW (2001) Multiphoton microscopy in biological research. Curr Opin Chem Biol 5:603-608. doi:10.1016/S1367-5931(00)00241-6

Xu C, Zipfel W, Shear JB, Williams RM, Webb WW (1996) Multiphoton fluorescence excitation: new spectral windows for biological nonlinear microscopy. Proc Natl Acad Sci USA 93:10763-10768. doi:10.1073/pnas.93.20.10763
Zipfel WR, Williams RM, Webb WW (2003) Nonlinear magic: multiphoton microscopy in the biosciences. Nat Biotechnol 21:1369-1377. doi:10.1038/nbt899

Zucchelli G, Jennings RC, Garlaschi FM (1992) Independent fluorescence emission of the chlorophyll spectral forms in higher plant photosystem II. Biochim Biophys Acta 1099:163-169 